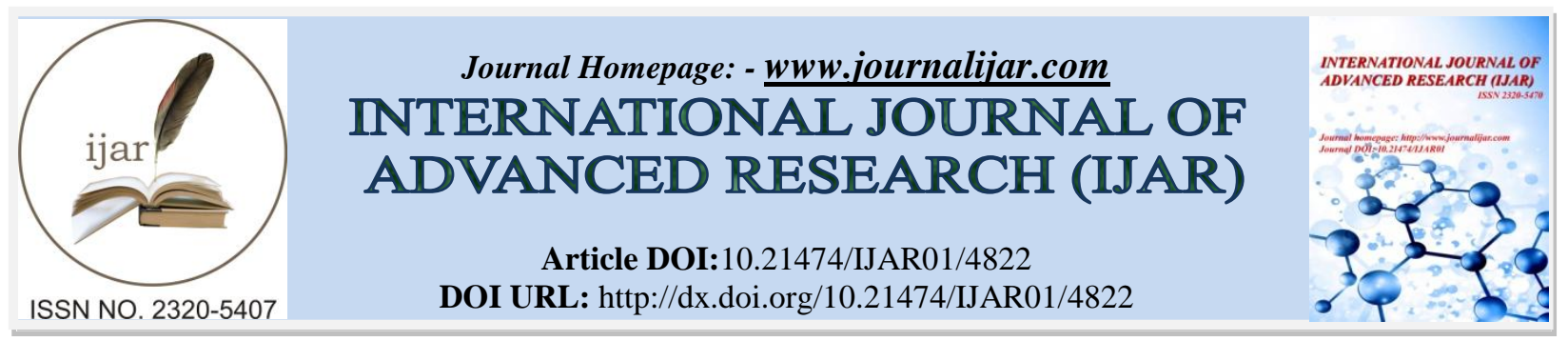

RESEARCH ARTICLE

\title{
SCREENING OF PHYTOCHEMICALS \& BIOACTIVE ANTIBACTERIAL ACTIVITY IN SPIROGYRA
}

$S P$.

\section{Prarthana $\mathbf{J}^{1}$ and Maruthi $\mathrm{KR}^{2}$.}

1. Research Scholar, PRIST University, Tamilnadu 613 403, working at Dept of PG Studies \& Research in Biotechnology, SDM (Autonomous college) Ujire 574240,Karnataka

2. Research Supervisor, PRIST University, Tamilnadu 613403.

\section{Manuscript Info}

\section{Manuscript History}

Received: 12 May 2017

Final Accepted: 14 June 2017

Published: July 2017

Key words:-

Algae, Spirogyra sp ,phytochemical, antibacterial activity, GC-MS, bioactive

\section{Abstract}

Algae are very important component of aquatic ecosystem, known for producing several biologically active compounds with antiviral, antibacterial, antifungal, and anticancer activities. In the present investigation, the effect of different solvents, including methanol, acetone, petroleum ether, benzene, chloroform, hexane, diethyl ether, ethanol, ethyl acetate were done on Spirogyra sp. Dried algal powder after identification were cold extracted \& screened for glycosides,alkaloids,saponins, flavanoids,tannins,terpenoids, phenolics, anthraquinones,cardiac glycosides, etc. The extracts showing good phenolic content were tested for antibacterial activity against pure cultures of fish pathogens namely Aeromonashydrophila,. The GCMS analysis of the extracts revealed the presence of several antimicrobial bioactive constituents in the extracts.

Copy Right, IJAR, 2017,. All rights reserved.

\section{Introduction:-}

Aquatic organisms are a rich source of structurally novel \& biologically active metabolites[8].Many microscopic forms of algae are known to grow in fresh water as slimy 'blanket weed'. One such familiar algae is Spirogyra a filamentous algae, its cells form each filament consists of an extensive chain of identical cells. Spirogyra is the most common genus of Zygnemataceae and member of the freshwater algae. It exhibits the greatest diversity of the 12 to 13 genera recognized in this family of green algae (Transeau 1951, Kadlubowska 1972).

Seasonal variation in $\mathrm{pH}$, temperature \& dissolved oxygen play an important role in the multiplication of pathogens leading to disease in fish [73].Aeromonashydrophila is an opportunistic pathogen known to cause disease during stressed condition.Symptoms of the disease may range from sudden death to lack of appetite, swimming abnormalities, pale gills \& skin ulceration. Current treatment involves use of antibiotics such as Tetramycin®, an oxytetracycline, and Remet-30®. Potential problems associated with antibiotic include inadequate dosage, overdosing, drug resistance by bacteria[74]

The use of algae for therapeutic purpose has a long history \& systemic examination of algae for biologically active substances. The aqueous \& solvent extracts from algae were tested against gram positive \& gram negative bacteria [7-10].The first investigation of the antibiotic activity of algae was carried out by (Pratt et al., 1944).Evidence of phytochemical and pharmacological studies on algae is available in the literature with special references to terpenoids and steroids (Parameswaran et al. 1944; Patterson et al 1968). Secondary and primary metabolites

Corresponding Author:-Prarthana J.

AddressDepartment of P.G. Studies \& Research in Biotechnology S.D.M. (Autonomous) College, Ujire- 
produced by these organisms may be potential bioactive compounds of interest in pharmaceutical industry. There are a number of reports on the evaluation of antioxidant activity in microalgae and cyanobacteria belonging to the genera Botryococcus (Rao et al. 2006), Chlorella (Wu et al. 2005; Goh et al.2010), Dunaliella(Herrero et al. 2006), Nostoc (Li et al.2007), Phaeodactylum(Guzman et al. 2001), Spirulina (Miranda et al. 1998; Jaime et al. 2005; Mendiola et al.2007), Haematococcus(Cerón et al. 2007) andChaetoceros (Goh et al. 2010). These studies concluded that several microalgal genera contain potent antioxidants, both from lipophilic and hydrophilic nature. Justella et al (2011)proved antimicrobial property of ethanolic extracts of Spirogyra grantianaagainstE.coli. Antimicrobial \& antioxidants activity of Euglena tuba, Oscillatoriaagardhii\&Anabenasphaerica,Cosmariumsp, Chlorococcumhumicola (Dipankar et al 2014;Abd El-Aty et al2014; Challouf et al2012;Bhagavathy et al 2011)In the present study dried algal extracts were dissolved in different solvents with the increasing order of polarity, different extracts were tested for the presence of chemical constituents. The extracts showing good phenolic content were estimated \& analyzed for antimicrobial activity against fish pathogens Aeromonashydrophila. GC-MS analysis of the Spirogyra Diethyl ether,Acetone\& Ethyl acetate extracts reported several bioactive components with antimicrobial property.

\section{Material and method:-}

\section{Sample preparation \& extraction:-}

Samples were collected from Arasinamakki, near Shishila. Dakshinakannada, India .Algae samples were cleaned all epiphytes and necrotic parts were removed. Samples were rinsed with sterile water to remove any associated debris. Sample was kept under sunshade for 7 days. After drying the sample, it was ground thoroughly to powder form. The powdered samples were extracted with at room temperature with different solvents. The extraction is carried out for 10 days, after that extracts were filtered, the filtrate is dried using rot evaporator and concentration is determined\& subjected to analysis. (Gonzalez del valet.al, 2001.)

\section{Phytochemical Analysis:-}

The extracts were subjected to phytochemical tests for presence of following biomolecules byUsing the standard qualitative procedures as described in literature [15].

1. Test for Glycosides: $10 \mathrm{ml}$ of $50 \% \mathrm{H} 2 \mathrm{SO} 4$ was added to the $1 \mathrm{ml}$ of extract in a boiling tube. The mixture was heated in boiling water bath for $5 \mathrm{~min} .10 \mathrm{ml}$ of Fehling's solution ( $5 \mathrm{ml}$ of each solution AandB) was added and boiled. A brick red precipitate indicated the presence of glycosides.

2. Test for Alkaloids: $1 \mathrm{ml}$ of $1 \% \mathrm{HCl}$ was added to the $3 \mathrm{ml}$ of extract in a test tube and was treated with few drops of Meyer's reagent. A creamy white precipitate indicated the presence of alkaloids.

3. Test for saponins: $5 \mathrm{ml}$ of extract was shaken vigorously to obtain a stable persistent froth. The frothing was then mixed with 3 drops of olive oil and observed for the formation of emulsion, which indicated the presence of saponins.

4. Test for flavonoids: A few drops of $1 \% \mathrm{NH} 3$ solution was added to the $2 \mathrm{ml}$ of extract in a test tube. A yellow coloration was observed for the presence of flavonoids.

5. Test for tannins: To $0.5 \mathrm{ml}$ of extract solution, $1 \mathrm{ml}$ of distilled water and 1-2 drops of ferric chloride solution were added and observed for brownish green ora blue black coloration.

6. Test for terpenoids: $5 \mathrm{ml}$ of extract was mixed with $2 \mathrm{ml}$ of $\mathrm{CHCl} 3$ in a test tube. $3 \mathrm{ml}$ of concentrated $\mathrm{H} 2 \mathrm{SO} 4$ was carefully added along the wall of the test tube to form a layer. An interface with a reddish brown coloration was confirmed the presence of terpenoids.

7. Test for cardiac glycosides: $5 \mathrm{ml}$ of extract was mixed with $2 \mathrm{ml}$ of glacialacetic acid containing $1 \mathrm{drop}$ of $\mathrm{FeCl3}$. Theabove mixture was carefully added to the $1 \mathrm{ml}$ of concentrated H2SO4. Presence of cardiacglycosides was detected by the formation of a brown ring.

8. Test for phlobatannins: $10 \mathrm{ml}$ of extract was boiled with $1 \% \mathrm{HCl}$ in a boiling tube. Deposition of a red precipitate indicated the presence of phlobatannins

9. Test for Anthraquinones: Extract was mixed well with benzene, and then half of its own volume of $10 \%$ ammonia solution was added. Presence of a pink, red or violet coloration in the ammonial phase indicated the anthraquinones.

10. Test for Phenols: $3 \mathrm{~mL}$ of $10 \%$ lead acetate solution were added to $5 \mathrm{~mL}$ of plant extract. A bulky white precipitates indicated the presence of phenols. 


\section{Estimation of Phenolic Content:-}

The amount of total phenolics[1] in methanol extract was determined with Folin-Ciocalteu reagent according to the method of Singleton and Rossi with Gallic acid as the standard [23]. Briefly standard stock solution of $10 \mathrm{mg} / 10 \mathrm{ml}$ of gallic acid was prepared in distilled water. From this, various concentrations ranging from $200-1000 \mu \mathrm{g} / \mathrm{ml}$ were prepared. To this $1 \mathrm{ml}$ Folin and Ciacalteau reagents (1:2 with water) was added and kept at room temperature for 5 min and then $1 \mathrm{ml}$ of $7 \%$ sodium carbonate solution was added to the reaction mixture and incubated at room temperature for 90 minutes. The colour developed was read at $750 \mathrm{~nm}$. A $100 \mu \mathrm{l}$ of each extract of sample was mixed with the same reagents. Gallic acid was used as the reference standard and the results are expressed as milligram gallic acid equivalent ( $\mathrm{mg} / \mathrm{g}$ dry weight of Spirogyra $\mathrm{sp}$ ).

\section{Antibacterial Activity:-}

Pure cultures of Aeromonashydrophila, was used as test micro-organisms. Different solvent extracts were checked for antibacterial activity against the lawn cultures by agar well diffusion method. In each respective solvent is chosen as in the form of control.

\section{Gas chromatography and mass spectrometry Analysis:-}

Gas chromatography-mass spectrometry (GC-MS) analysis was performed using an BR-5MS(5\%Diphenyl/95\% Dimethyl poly siloxane)capillary column (length $30 \mathrm{~m} \times$ diameter $0.25 \mathrm{~mm} \times$ film thickness $0.25 \mu \mathrm{m}$ ) with helium at $1 \mathrm{ml}$ for $1 \mathrm{~min}$ as a carrier gas. The mass spectrometer was operated in the electron impact (El) mode at $70 \mathrm{eV}$ in the scan range of 50-500 m/z. The split ratio was adjusted to $1: 10$, and the injected volume was $2 \mu$. The injector temperature was $280{ }^{\circ} \mathrm{C}$, and the oven temperature was kept at $110{ }^{\circ} \mathrm{C}$ for $3.5 \mathrm{~min}$, rose to $280{ }^{\circ} \mathrm{C}$ at $5{ }^{\circ} \mathrm{C} \mathrm{min}^{-1}$ (total run time $37.50 \mathrm{~min}$ ). Peak identification of crude spirogyra extracts were performed by comparison with retention times of standards, and the mass spectra obtained were compared with those available in NIST libraries (NIST 11 - Mass Spectral Library, 2011 version) with an acceptance criterion of a match above a critical factor of $80 \%$ according to Srinivasan et al 2016

\section{Results and Discussion:-}

\section{Phytochemical Analysis:-}

Important phytochemicals, such as alkaloids, triterpenoids, steroids, tannin, saponin, coumarins, terpenoids, quinine, phytosteroids, phlobatannins and flavonoids were screened for their presence and presented in Table 1.

Table1:-Phytochemical constituents of Spirogyra extracts.

\begin{tabular}{|c|c|c|c|c|c|c|c|c|c|}
\hline Spirogyra sp. & $\begin{array}{l}\text { Ethano } \\
1 . \\
\text { Extract }\end{array}$ & $\begin{array}{l}\text { Aceton } \\
\mathrm{e} \\
\text {.Extrac } \\
\mathrm{t}\end{array}$ & $\begin{array}{l}\text { Methano } \\
1 . \\
\text { Extract }\end{array}$ & $\begin{array}{l}\text { DiEthylEth } \\
\text { er. Extract }\end{array}$ & $\begin{array}{l}\text { Benzen } \\
\text { e. } \\
\text { Extract }\end{array}$ & $\begin{array}{l}\text { Chlorofor } \\
\text { m. Extract }\end{array}$ & $\begin{array}{l}\text { Hexan } \\
\text { e. } \\
\text { Extract }\end{array}$ & $\begin{array}{l}\text { Petroleu } \\
\text { m ether. } \\
\text { Extract }\end{array}$ & $\begin{array}{l}\text { Ethyl } \\
\text { Acetat } \\
\text { e. } \\
\text { Extract }\end{array}$ \\
\hline Glycosides & -- & -- & -- & -- & -- & -- & -- & -- & -- \\
\hline Alkaloids: & -- & -- & -- & -- & ++ & -- & -- & -- & -- \\
\hline Spooning & ++ & ++ & ++ & -- & -- & -- & -- & -- & -- \\
\hline Flavonoids & -- & ++ & -- & ++ & -- & -- & -- & -- & ++ \\
\hline Tannins & -- & ++ & -- & ++ & + & -- & -- & ++ & ++ \\
\hline Terpenoids & -- & ++ & ++ & -- & ++ & ++ & & ++ & ++ \\
\hline $\begin{array}{l}\text { cardiac } \\
\text { glycosides }\end{array}$ & ++ & ++ & ++ & ++ & ++ & ++ & ++ & -- & ++ \\
\hline $\begin{array}{l}\text { Phlobatannin } \\
\text { s }\end{array}$ & -- & -- & -- & -- & -- & -- & -- & -- & -- \\
\hline $\begin{array}{l}\text { Anthraquinon } \\
\text { es }\end{array}$ & -- & -- & -- & -- & -- & -- & -- & -- & -- \\
\hline Phenols & ++ & ++ & ++ & -- & -- & -- & ++ & -- & -- \\
\hline Sterols & -- & ++ & -- & -- & -- & ++ & -- & -- & ++ \\
\hline Resins & -- & -- & -- & -- & -- & -- & -- & -- & -- \\
\hline
\end{tabular}

++ Copiously present, + moderately present, - absent 


\section{Estmation of Phenolic content:-}

Diethyl ether, Acetone \& Ethyl acetate spirogyra extracts shown positive for tannins, flavonoids,terpenoids are analyzed .Highest phenolic content shown in ethyl acetate extract Table 2

Table2:- Assay of Phenolic content of Spirogyra Acetone,Diethyl ether \& Ethyl acetate extracts

\begin{tabular}{|l|l|l|l|l|}
\hline Sl.No & StdConc & OD 750nm & Sample extract $(0.1 \mathrm{ml})$ & OD at 750nm \\
\hline 1 & $0.2 \mathrm{mg}$ & 0.24 & Acetone & 0.63 \\
\hline 2 & 0.4 & 0.41 & DEE & 0.04 \\
\hline 3 & 0.6 & 0.90 & Eth Ace & 1.09 \\
\hline 4 & 0.8 & 1.22 & \multicolumn{2}{|l}{} \\
\cline { 1 - 3 } 5 & 1.0 & 1.95 & & \\
\cline { 1 - 2 }
\end{tabular}

Antibacterial activity:-

The antibacterial activity of Spirogyra extracts of Diethyl ether, Acetone \& Ethyl acetate on Aeromonashydrophila presented in Table 3.The agar well diffusion method was used to evaluate the antibacterial activity by measuring the zone of inhibition. Among three extracts Spirogyra ethyl acetate extract was found to be superior controlling growth of all three pathogens.

Table 3:- Antibacterial activity of extracts depicted through zone of inhibition

\begin{tabular}{|l|l|l|l|l|l|l|}
\hline Sl.No & & \multicolumn{5}{|c|}{ Zone of inhibition in mm } \\
\hline \multirow{2}{*}{1} & \multirow{2}{*}{ Sample Extract } & \multicolumn{5}{|c|}{ Aeromashydrophila } \\
\cline { 3 - 7 } & & $10 \mu l$ & $25 \mu l$ & $50 \mu l$ & $100 \mu l$ & $C \mu l$ \\
\hline 2 & DiEthylEther & -- & -- & $10 \pm 0.11$ & $11 \pm 0.32$ & $08 \pm 0.43$ \\
\hline 3 & Acetone & -- & $11 \pm 0.21$ & $12 \pm 0.33$ & $12 \pm 0.55$ & $10 \pm 0.54$ \\
\hline 4 & Ethyl Acetate & $15 \pm 0.56$ & $18 \pm 0.41$ & $23 \pm 0.33$ & $26 \pm 0.57$ & $13 \pm 0.62$ \\
\hline
\end{tabular}

Values are mean inhibition zone $(\mathrm{mm}) \pm$ S.D of three replicate

MIC for Diethyl ether $5.54 \mathrm{mg} / \mathrm{ml}$, MIC for Acetone extract $3.75 \mathrm{mg} / \mathrm{ml}$, MIC Ethyl acetate $2.15 \mathrm{mg} / \mathrm{ml}$

GC-MS Analysis:-

The GC-MS analysis of the crude Spirogyra Diethyl ether extract, Table 4 revealed seven components of that the main chemical constituent was the Bus(2-ethylhexyl)phthalate Retention time(RT)23.94min ,92.54\%\&nHexadecanooic acid RT $15.61 \& 2.35 \%$. Acetone extract,Table 5 revealed twenty four peaks of that main peaks were n-Hexadecanoic acid RT $15.82 \& 29.02 \%$,Bis(2-ethylhexyl)phthalate RT23.88 \& 21.60\%, Tetradecanoic acid RT13.13 \& $16.46 \% \& O c t a d e c e n o i c ~ a c i d R T 18.50 \& 11.17 \%$. Ethylacetateextracts Table 6showed thirty six peaks of that main peaks were Bis(2-ethylhexyl)phthalate RT $23.97 \& 65.86 \%$, n-Hexadecanoic acid RT $15.88 \& 10.81 \%$.

Table 4:- GC MS analysis of Spirogyra Diethyl ether extracts

\begin{tabular}{|c|c|c|c|c|}
\hline Name of the compound & RT & Mol.Wt & Peak Area\% & \\
\hline ButylatedHyydroxytoluene & 9.98 & 220 & 1.36 & $\begin{array}{c}\text { Antioxidant } \\
\text { Antimutagenic, anticarcinogenic,inactivation of } \\
\text { virus[28] }\end{array}$ \\
\hline Methoprene & 10.54 & 310 & 0.08 & Insectisidal[29] \\
\hline Tetradecanoic acid & 13.08 & 228 & 1.93 & Antibacterial \& antifungal[30] \\
\hline Dibutyl phthalate & 15.55 & 278 & 1.56 & $\begin{array}{l}\text { Antimicrobial,Disruptor of estrogen activity } \\
{[31]}\end{array}$ \\
\hline n-Hexadecanooic acid & 15.62 & 256 & 2.35 & $\begin{array}{c}\text { Antioxidant, hypocholesterolemic, } \\
\text { anti-inflammatory } \\
\text {, antibacterial[32] }\end{array}$ \\
\hline Octadecanoic acid & 18.44 & 284 & 0.19 & $\begin{array}{c}\text { Cancer preventive } \\
\text { Insectifuge* }\end{array}$ \\
\hline Bis(2-ethylhexyl)phthalate & 23.94 & 390 & 92.54 & Antibacterial \& antifungal[33] \\
\hline
\end{tabular}


Table 5:- GC MS analysis of Spirogyra Acetone extracts

\begin{tabular}{|c|c|c|c|c|}
\hline Name of the compound & RT & $\begin{array}{l}\text { Mol. } \\
\text { Wt }\end{array}$ & Peak Area\% & \\
\hline Octanoic acid & 4.98 & 144 & 0.02 & Fungicide,Pesticide,Candidicide* \\
\hline Nonanoic acid & 6.47 & 158 & 0.32 & Herbicide[34] \\
\hline $\begin{array}{l}\text { 2H-Pyran-2-one,tetrahydro-4- } \\
\text { hydroxy-6-pentyl }\end{array}$ & 8.22 & 186 & 0.34 & Antimicrobial \&anticancerous[35] \\
\hline Undecylenic acid & 9.09 & 184 & 0.46 & Not reported \\
\hline 12-Hydroxydodecanoic acid & 9.63 & 216 & 0.84 & $\begin{array}{l}\text { Antimicrobial,antioxidant,antiallergic, antiaging } \\
\text {,antitumor,anti-inflammatory[36] }\end{array}$ \\
\hline $\begin{array}{l}\text { 2(4H)-Benzofuran,5,6,7,7a- } \\
\text { tetrahydro-4,4,7a-trimethyl }\end{array}$ & 10.43 & 180 & 1.50 & Antimicrobial[37] \\
\hline Dodecanoic acid & 10.71 & 200 & 2.16 & Not reported \\
\hline Dihydrojasmone & 11.21 & 166 & 0.99 & Antimicrobial \& antifungal[38] \\
\hline Azelaic acid & 11.81 & 188 & 1.43 & Antimicrobial[39] \\
\hline Tetradecanoic acid & 13.13 & 228 & 16.46 & Antimicrobial \& anti-inflammatory[40] \\
\hline $\begin{array}{l}9,10- \\
\text { Dimethyltricyclo[4.2.1.1(2,5)]d } \\
\text { ecane-9,10-diol }\end{array}$ & 13.30 & 196 & 1.91 & Anticancerous[41] \\
\hline $\begin{array}{l}\text { 2-Pentadecanone, } 6,10,14- \\
\text { trimethyl }\end{array}$ & 14.07 & 268 & 2.26 & Repellent to arthropod[42] \\
\hline Pentadecanoic acid & 14.32 & 242 & 2.55 & Antioxidant, antibacterial[43] \\
\hline $\begin{array}{l}\text { 2H-Pyran-2-one,tetrahydro-6- } \\
\text { nonyl }\end{array}$ & 14.83 & 226 & 0.23 & Not reported \\
\hline n-Hexadecanoic acid & 15.82 & 256 & 29.02 & Antiinflammatory[44] \\
\hline $\begin{array}{l}\text { 11,13-Dimethyl-12-tetradecen- } \\
\text { 1-ol acetate }\end{array}$ & 16.61 & 282 & 0.82 & Antioxidant \&antitumour activity[45] \\
\hline Trans-13-Octadecenoic acid & 18.10 & 282 & 2.70 & Antimicrobial[46] \\
\hline Octadecenoic acid & 18.50 & 284 & 11.17 & Free radical scavenging activity[47] \\
\hline Erucic acid & 19.07 & 338 & 1.23 & Not reported \\
\hline $\begin{array}{l}\text { z)-3,7,11-Trimethyldodec-2- } \\
\text { enoic acid,methyl ester }\end{array}$ & 19.93 & 254 & 0.34 & Not reported \\
\hline $\begin{array}{l}\text { 7-Methyl-Z-tetradecen-1-ol } \\
\text { acetate }\end{array}$ & 21.44 & 268 & 1.03 & $\begin{array}{l}\text { Anticancer,anti- } \\
\text { inflammatory,hepatoprotective[48] }\end{array}$ \\
\hline Cis-11-Eicosenoic acid & 21.85 & 310 & 0.26 & Not reported \\
\hline $\begin{array}{c}\text { Tricyclo[20.8.0.0(7,16)]triacont } \\
\text { ane,1(22),7(16)- } \\
\text { diepoxy } \\
\end{array}$ & 22.74 & 444 & 0.37 & Antidiabetic type II[49] \\
\hline Bis(2-ethylhexyl)phthalate & 23.88 & 390 & 21.60 & Antibacterial \& antifungal[33] \\
\hline
\end{tabular}

*Dr.Duke'sphytochemicals\&ethanobotanical databases

Table 6:- GC MS analysis of Spirogyra Ethyl acetate extracts

\begin{tabular}{|l|l|l|l|l|}
\hline Name of the compound & RT & Mol.Wt & Peak Area\% & \\
\hline Nonanoic acid & 6.93 & 158 & 0.10 & Herbicide[34] \\
\hline Diethyl adipate & 8.23 & 202 & 0.34 & Antimicrobial anti inflammatory[50] \\
\hline Ethyl 5-methylhexonate & 8.51 & 158 & 0.00 & \\
\hline Diethyl pimelate & 9.65 & 216 & 0.62 & Antioxidant \& antimicrobial[51] \\
\hline $\begin{array}{l}\text { Nonanoic acid,9-oxo-,ethyl } \\
\text { ester }\end{array}$ & 9.94 & 200 & 0.55 & $\begin{array}{l}\text { Antioxidant,anti inflammatory, } \\
\text { anticancerous[52] }\end{array}$ \\
\hline Ethyl hydrogen suberate & 10.63 & 202 & 0.16 & Antioxidant anti inflammatory[53] \\
\hline Diethyl suberate & 10.96 & 230 & 0.89 & Antifungal ,antibacterial[54] \\
\hline $\begin{array}{l}\text { 3-Cyclopentylpropionic } \\
\text { acid,ethyl ester }\end{array}$ & 11.12 & 170 & 0.18 & Antibacterial[55] \\
\hline
\end{tabular}




\begin{tabular}{|c|c|c|c|c|}
\hline $\begin{array}{l}\text { Nonanedioicacid,monomethyl } \\
\text { ester }\end{array}$ & 11.87 & 202 & 0.66 & Antitumour [56]. \\
\hline $\begin{array}{l}\text { Nonanedioicacid,dimethyl } \\
\text { ester }\end{array}$ & 12.16 & 216 & 1.98 & Antimicrobial,anti inflammatory[57] \\
\hline $\begin{array}{l}\text { 12-Oxododecanoic acid,ethyl } \\
\text { ester }\end{array}$ & 12.43 & 242 & 0.05 & Antiseptic , antimicrobial,anti oxidant[58] \\
\hline $\begin{array}{l}\text { Thiazolo[3,2-a]pyrimidin-5- } \\
\text { one,7-methyl-2,3-dihydro- }\end{array}$ & 12.57 & 168 & 1.60 & Antibacterial , antibiofilm[59] \\
\hline Tetradecanoic acid & 13.17 & 228 & 4.87 & No report \\
\hline Decanedioic acid & 13.34 & 258 & 0.21 & No report \\
\hline Tetradecanoicacid,ethyl ester & 13.45 & 256 & 1.92 & Anticncerous[60] \\
\hline $\begin{array}{l}\text { 2-Pentadecanone, } 6,10,14- \\
\text { trimethyl- }\end{array}$ & 14.08 & 268 & 0.93 & Not reported \\
\hline Pentadecanoic acid & 14.23 & 242 & 0.27 & Antimicrobial[61] \\
\hline $\begin{array}{l}\text { Ethyl } 13 \text {-methyl- } \\
\text { tetradeconate }\end{array}$ & 14.33 & 270 & 0.36 & Antibacterial[62] \\
\hline $\begin{array}{l}\text { Dodecanedioicacid,dimethyl } \\
\text { ester }\end{array}$ & 14.59 & 258 & 0.26 & Antibacterial,antimicrobial,antidiabetic[63] \\
\hline $\begin{array}{l}\text { Hexadecanoicacid,methyl } \\
\text { ester }\end{array}$ & 15.20 & 270 & 0.13 & Antimicrobial anti inflammatory[64] \\
\hline n- Hexadecanoic acid & 15.88 & 256 & 10.81 & Antiinflammatory[44] \\
\hline Hexadecanoicacid,ethyl ester & 16.11 & 284 & 1.15 & Antimicrobial,anti oxidant [58] \\
\hline Octadecanoic acid & 18.53 & 284 & 1.79 & Free radical scavenging activity[47] \\
\hline $\begin{array}{l}\text { Octadecanoic acid,17-methyl- } \\
\text {,methyl ester }\end{array}$ & 18.91 & 312 & 0.33 & Antioxidant,antimicrobial[65] \\
\hline $\begin{array}{l}\text { 3-Buten-2-one,4-(3-hydroxy- } \\
\text { 6,6-dimethyl-2- } \\
\text { methylenecyclohexyl)- }\end{array}$ & 19.24 & 208 & 0.34 & No report \\
\hline $\begin{array}{l}\text { Z)-3,7,11-Trimethyldodec-2- } \\
\text { enoic acid,methylester }\end{array}$ & 19.83 & 254 & 0.21 & Insecticidal[66] \\
\hline $\begin{array}{l}\text { Cholest-22-ene-21-ol,3,5- } \\
\text { dehydro-6-methoxy-,pivalate }\end{array}$ & 20.76 & 498 & 0.68 & Anticancerous, antiviral \\
\hline $\begin{array}{l}\text { Octadecanoic acid,10-oxo- } \\
\text {,methyl ester }\end{array}$ & 21.53 & 312 & 0.38 & No report \\
\hline $\begin{array}{l}\text { (E)-9-Octadecenoic acid ethyl } \\
\text { ester }\end{array}$ & 21.83 & 310 & 1.04 & No report \\
\hline $\begin{array}{l}\text { i-Propyl 11,12-methylene - } \\
\text { octadecanoate }\end{array}$ & 22.23 & 338 & 0.16 & Antimicrobial[68] \\
\hline Ethyl Olelate & 22.57 & 310 & 0.36 & Flavour[69]. \\
\hline Bis(2-ethylhexyl)phthalate & 23.97 & 390 & 65.86 & Antibacterial \& antifungal[33] \\
\hline Docosanoic acid ,ethyl ester & 24.82 & 368 & 0.16 & No report \\
\hline $\begin{array}{l}\text { Eicosanoic acid,2- } \\
\text { (acetyloxy)-1- } \\
\text { [(acetyloxy)methyl]ethyl ster }\end{array}$ & 25.03 & 470 & 0.08 & Antibacterial[70]antioxidants [71] \\
\hline Eicosanoicacid,ethyl ester & 27.61 & 340 & 0.56 & No report \\
\hline $\begin{array}{l}\text { Cholestan-3-ol,2-methylene- } \\
,(3 \beta, 5 \alpha) \text { - }\end{array}$ & 28.46 & 400 & 0.01 & Antioxidant[72] \\
\hline
\end{tabular}

RT: Retention time, Molwt: Molecular weight

\section{Conclusion:-}

Aeromonashydrophila have the potential of causing zoonotic disease, a disease spreads from animal to human being during accidental cases. Currently antibiotic treatment is preferred to resolve disease. But, use of antibiotics has 
potential problem of being include inadequate dosage, overdosing, drug resistance by bacteria. Hence in the present study natural antimicrobial substance as a substitute for synthetic antibiotics is analyzed. Spirogyra extracts are prepared in different solvents with increasing order of polarity. Extracts are subjected to phytochemical tests for Glycosides,alkaloids, saponins,flavonoids,tannins,phenols,cardiac glycosides, sterols, resins etc. Extracts showing positive for phenol, tannins, flavonoids were estimated for phenolic content \& tested for antimicrobial activity against pure cultures of Aeromonashydrophila. Crude extracts subjected to GC -MS analysis reported many several bioactive compound showing antimicrobial, antioxidant\&anti fungal property. The cold extraction procedure adopted helped in the accountability of lipidous\& hydrocarbon molecule. Such natural antimicrobial substance showing broad spectrum activity can be used to replace synthetic antibiotics more effectively, less toxicity also can development of antibiotic resistant strains can be curtailed.

\section{Acknowledgement:-}

Author would like to thank ShriDharmasthalaManjunatheshwaraCollege(Autonomous) for providing laboratory ambience to carry out work.

\section{References:-}

1. V.L. Singleton and J.A. Rossi, Colorimetry of total phenolics with phosphomolybdic- phosphotungstic acid reagents, Am. J. of Enol. Viticu. 6 (1965) 144-158.

2. Ely R, Supriya T, Naik CG. Antimicrobial activity of marineorganisms collected off the coast of South East India. J ExpMarBiol Ecol2004; 309: 121-127.

3. Krishnaraju AV, Rao TVN, Sundararaju D, Vanisree M, TsayHS,Subbaraju GV. Assessment of bioactivity of Indian medicinalplants using brine shrimp (Artemiasalina) lethality assay.IntJApplSciEng2005; 2: 125-134.

4. Raghavendra MP, Satish S, Raveesha KA. Phytochemicalanalysis and antibacterial activity of Oxalis corniculata, a knownmedicinalplant.My Sci2006; 1(1): $72-78$.

5. Selvamaleeswaran P, Wesely EG, Johnson M, VelusamyS,Jeyakumar N. The effect of leaves extracts of ClitoriaternateaLinn against the fish pathogens. Asian Pac J Trop Med2010; 3(9):723-726.

6. Haripriya D, Selvan N, Jeyakumar N, Periasamy RS, Johnson M,rudayaraj V. The effect of extracts of SelaginellainvolvensandSelaginellainaequalifolialeaves on poultry pathogens. Asian PacJ Trop Med2010; 3(9): 678-681.

7. Edeoga HO, Okwu DE, Mbaebie BO. Phytochemical constituentsof some Nigerian medicinal plants.Afr $J$ Biotechnol2005; 4:685-688.

8. Prashanthkumar P, Angadi SB, Vidyasagar GM. Antimicrobialactivity of blue green algae and green algae. Indian J Pharm Sci2006; 88(6): 647-648.

9. Singh AP, Chaudhary BK. Preliminary phytochemical analysisandin vitro anti-bacterial activity screening of Pithosphoraoedogonia(Mont.) Wittrock-a fresh water green alga forming matsin the water bodies. J Algal Biomass Util2010; 1(2): 33-41.

10. Goud MJP, Seshikala D, CharyaMAS.Antibacterial activity andbiomolecular composition of certain fresh water microalgae fromRiver Godavari (India).Sci World J 2007; 2(3): 19-23.

11. Marhivanan K, Ramamurthy V, Rajaram R. Antimicrobial activityofOscillatoria princes and Lyngbya majuscule against pathogenicmicrobes. Int J Curr Res2010; 5: 97-101.

12. Parekh J, Chanda VS. In vitro antimicrobial activity andphytochemical analysis of some Indian medicinal plants.Turk JBiol2007; 31: 53-58.

13. Transeau, E. N. (1951). The Zjgnemataceae.The Ohio State University Press, Columbus, Ohio, pp.327.

14. Kadłubowska, J. Z. (1972). Chlorophyta V. Conjugales: Zygnemaceae. Zrostnicowate. In: Starmach K, Siemin'skaJ(eds) Flora słodkowodnaPolski, 12A. PWN, Krako'w, pp 431.

15. Goh S-H, Yusoff FM, Loh S-P (2010) A comparison of the antioxidant properties and total phenolic content in a diatom, Chaetoceros sp. and a green microalga, Nannochloropsis sp. J AgrSci 2:123-130

16. Guzman S, Gato A, Galleja JM (2001) Antiinflammatory, analgesic and free radical scavenging activities of the marine microalgae Chlorella stigmatophora and Phaeodactylumtricornutum. Phytother Res 15:224-230

17. Li H, Cheng K, Wong C, Fan K, Chen F, Jiang Y (2007) Evaluation of antioxidant capacity and total phenolic content of different fractions of selected microalgae. Food Chem 102:771-776

18. Miranda MS, Cintra RG, Barros SB, Mancini Filho J (1998) Antioxidant activity of the microalga Spirulina maxima. Braz J Med Biol Res 31:1075-1079

19. Mendiola J, Jaime L, Santoyo S, Reglero G, Cifuentes A, Ibanez E et al (2007) Screening of functional compounds in supercritical fluid extracts from Spirulinaplatensis. Food Chem 102:1357-1367 
20. Cerón MC, García-Malea MC, Rivas J, Acien FG, Fernandez JM, Del Río E, Guerrero MG, Molina E (2007) Antioxidant activity of Haematococcuspluvialis cells grown in continuous culture as a function of their carotenoid and fatty acid content.ApplMicrobiolBiot 74:1112-1119

21. Rao AR, Sarada R, Baskaran V, Ravishankar GA (2006) Antioxidant activityofBotryococcusbrauniiextractelucidatedinvitromodels.JAgric Food Chem 54:4593-4599

22. Jaime L, Mendiola JA, Herrero M, Soler-Rivas C, Santoyo S, Señorans FJ, Cifuentes A, Ibáñez E (2005) Separation and characterization of antioxidants from Spirulinaplatensis microalga combining pressurized liquid extraction, TLC, and HPLC-DAD. J Sep Sci 28:2111-2119

23. Herrero M, Jaime L, Martín-Alvarez PJ, Cifuentes A, Ibáñez E (2006) Optimization of the extraction of antioxidants from Dunaliellasalina microalga by pressurized liquids. J Agric Food Chem 54:5597-5603

24. Singleton V.L., Rossi J.A., Colorimetry of total phenolics with phosphomolybdic - phosphotungstic acid reagents. Am. J. Enol. Vitic., 1965, 16, 144-158.

25. Parameswaran, P. S., Bhat, K. L., Das, B. N. \&Kamat, S. Y. 1944. Halogenated terpenoids from the brown alga Padinatetrastromatica (Hauck). Indian Journal of chemistry. 33: 1006-1008.

26. Patterson, G. W. 1968. Sterols of Laminaria.Comparative Biochemistry and Physiology. 24:501-505.

27. Pratt, R., Daniel, T. C., Eier, J.B., Gunnison, J. B., Kumler, W. D., Oneto, J.F., Strait, L. A., Spoehr, H. A., Hardin, G. J., Milner, H. W., Smith, H. \& Strain, H. H. 1944. Chlorellin.An antibacterial substance from chlorella.Science. 99:351-352.

28. GonzalezdelValA,PlatasG,BasilioA(2001).Screeningofantimicrobialactivitiesinred,greenandbrownmacroalgaefr omGranCanaria(CanaryIslands,Spain).Int.Microbiol.4:35-40.

29. [28]Snipes, W., Person, S., Keith, A. and Cupp, J. (1975) Science 188, 64-66. Brugh, M., jr (1977) Science 197, 1291-1292. Calle, L.M., Sullivan, P.D., Nettleman, M.D., Ocasio, I.J., Blazyk, J. and Jollick, J. (1978) Biothem.Biophys. Res. Commun. 85, 351-356. Black, H.S., Chan, J.T. and Brown, G.E. (1978) Cancer Res. 38, 1384-1387. McCay, P.B., King,M.M. and Pitha, J.V. (1981) Cancer Res. 41, 3745-3748. Slaga, T.J. and Bracken, W.M. (1977) Cancer Res. 37, 1631-1635. P)

30. Antunes-Kenyon, Steven; Kennedy, Gerard.; Massachusetts. Pesticide Bureau.Methoprene: A review of the impacts of the insect growth regulator methoprene on non-target aquatic organisms in fish bearing water(Massachusetts Pesticide Bureau, Department of Food and Agriculture, 2001-08).

31. AgoramoorthyG,ChandrasekaranM,VenkatesaluV, Antibacterial and

32. antifungal activities of fatty acid methyl esters of the blind-your-eye mangrove from India,Brazil $\mathrm{J}$ Microbiol, 2007; 38: 739-742.

33. R.N. Roy, S. Laskar, Dibutyl phthalate, the bioactive compound produced by Streptomyces albidoflavus 321.2 Microbiological Research 2006 Volume 161, Issue 2, Pages 121-126

34. Sermakkani M, Thangapandian V, gc-ms analysis of Cassiaitalica leaf methanolextract, Asian J Pharm Clin Res, 2012; 5(2):90-94

35. A. Kavitha, P. Prabhakar, M. VijayalakshmiandVenkateswarluProduction of bioactive metabolites by Nocardialevis MK-VL_113 Article first published online: Jul 2009

36. Franck E. Dayan and Stephen O. Duke,Natural Compounds as Next Generation Herbicides,2014,Plant Physiology Preview.

37. K.Ajaykumar., et al Journal of chemical \& pharmaceuticals,2015;Pyrans:Heterocycles of chemical \& biological interest.7(11):693-700

38. Pavel C et al,Biologicalactivitiesof Royal Jelly;Review;Animal sciences \& Biotechnologies,2011,44(2)

39. Farina Mujeeb et al,Phytochemical evaluation, antimicrobial activity \& determination of bioactive components from leaves of Aeglemarmelos, Biomed Research Institute, 2014

40. http://books.google.co.in ,http://www.jstage.jst.go.jp ,www.fisher.co.uk

41. BOJAR, R. A., HOLLAND, K. T. \&CUNLIFFE, W. J. (1991). The in vitro antimicrobial efTects of azelaic acid upon Propionibacterium acnes strain P37. Journal of Antimicrobial Chemotherapy 28, 843853.

42. www.hindawi.com/journals/bmri

43. Asma M. Alenad, Nabila A. Al-Jaber, SoundararajanKrishnaswamy, Sobhy M. Yakout,Nasser M. Al-Daghri and Majed S. Alokail.chilleafragrantissima extract exerts its anticancer effect via induction of differentiation, cell cyclearrest and apoptosis in chronic myeloid leukemia(CML) cell line K562 .Journal of Medicinal Plants Research ,Vol. 7(21), pp. 1561-1567, 3 June, 2013

44. Raju K Chalannavar et al .Chemical constituent of essential oil from Syzygiumcordatum(Mystaceae).African Journal of Biotechnology.2011.Vol.10(14)pg 2741-2745 
45. Dinesh C Sharma et al .Phytochemical evaluation,antioxidantassay, antibacterial activity \& determination of cell viability (J774 \& THP1 alpha cell lines)of P.Sylvestris leaf crude \& methanol purified fraction. EXCLI Journal 2016;15;85-94

46. vasudevanAparna,Kalarickal V Dileep,Pradeep K Mandal et al.,Anti inflammatory property of n-Hexadecanoic acid :Structural evidence \& kinetic assessment .Chemical Biology \& Drug design.Vol 80(3)434-439

47. Huang chun Yan et al Evaluation of antioxidant \&antitumour activity of lemon essential oil.Journal of medicinal plant research. 2010,vol4(8)1910-1915

48. Mustapha N. Abubakar and Runner R. T. Majinda .GC-MS analysis \&preliminaryantimicrobial activity of Albiziaadianthifolia(Schumach)and pterocapusangolensis(DC), Medicines 2016, 3, 3;

49. ]www.ncbi.nlm.nih.gov/pmc/articles/PMC3867356

50. www.academicjournals.org/article

51. www.springer.com/10.1186\%2F513588

52. Nehalsinghal et al ;Journal of chemical \&pharmaceuticalreseaarch ;2011,3(2):126-133;Recent Advancement of Triazole derivatives \& their biological significance.

53. PremJanes et al,Antimicrobial activity of Diethyl pimelate an insilicoapproach;Journal of pharmaceutical clinical research 7(4)2014

54. NishaSetal :Research Journal of pharmaceutical biological \& chemical sciences ISSN 09758585;Phytochemicalscreening \&GC MS analysis ofRhizomes of Marantaorundinacea.L

55. Vidhyamadhavi et al, Research journal of pharmaceutical biological sciences ISSN 0975-8585;Evaluation of invitro antioxidant,anti inflammatory properties of aerial parts of Zanthoxylumrhesta

56. Microbiological Research vol170;2015pg 213-222;Identification \& antifungal activity of novel organic compounds found in cuitcalar\& internal lipids of medicinally important files

57. A U Isakhamyan et al :Pharmacuetical chemistry Journal 47(9),2013:Synthesis \& some biological properties of amino alkyl ester methylioolides of substituted acetic \& propionic acids

58. FarzinH ,IJBS2007,3(1)60-64.Synthesis \&antitumour activity of substituted succinamides using a potato disc tumour induction assay

59. Sotifa A;2012,4(2);1281-1287 chem composition \& antimicrobial activity of essential oil \& lipid content of Cardeuspyenocephalus L. growing in Saudi arabia

60. Sudha T et al,Journal of Applied pharmaceutical sciencesvol3(5)pg;126-130,2013;GC MS analysis of bioactive components of aerial parts of FluggeaLeycopyrus(wild)

61. Dan zhao et a,Europen journal of Medicinal chemistry vol 87,2014pg 500-507-Biological evaluation of halogenate thiazole[3,2-a]pyrimidin-3-one,carboxcylic acid derivatives argeting the YycGHistine kinase

62. Meng-Fei Li et al electronic Journal of Biology,2009,vol5(1)11-16; The dormancy mechanism \&bioactivity of hydroquinone extracted from Podophyllumhexandrumroyle seed

63. Rajashri R Nai;OrientalJoournal of Chemistry vol 31 ;GC -FID analysis of fatty acids 7 biological activity of Zanthoxylumrhesta seed oil

64. Allazaablotskaya et al ;Silyl modification of biologically active compounds-13.synthesis,cytotoxicity \& antibacterial action of N-methyl-N(2-triorg-anylsiloxyethyl)-1,2,3,4-tetrahydro(iso)quinolinium iodies;Applied organometallic chemistry

65. Shodhganga.inflibnet.ac.in/chapter V;Synthesis Characterization \& biological activity of novel pyrazoline derivative

66. G Rajeshwari et al Reseaarch journal of pharmaceutical biological \&chemical sciencesISSN 0975-8585GC-MS analysis of bioactive components of Hugoniamystax.L.

67. Vigisaral ED et al,journal of chemical \& pharmaceutical Research 20146(8)294-300;GC-MS Analysis ofbioacive constituents of Indigoferasuffruticosa leaves

68. DenisaLiszeková, MajaPolakovičová, Milan Beňo, Robert Farkaš.Molecular Determinants of Juvenile Hormone Action as Revealed by 3D QSAR Analysis in Drosophila;PLOS;2009

69. MAH Nagalakshmi, K Sri Rama Murthy. Phytochemical Profile of Crude Seed Oil of Wrightiatinctoria R.BR. andWrightiaarborea(DENNST.) MABB. by GC-MS Int. J. Pharm. Sci. Rev. , 31(2), March 2015

70. ImadHadiHameed. Analysis of bioactive chemical compounds of Aspergillusnigerby using gas chromatography-mass spectrometry and fourier-transform infrared spectroscopy Vol. 7(8), pp. 132-163, August 2015 DOI: 10.5897/JPP2015.0354 Article Number: 5F9D8B754311

71. Lawrence, B.M., Essential Oils 1976-1977, Essential Oils 1978, Essential Oils 1979-1980

72. Mishra PravatManjari, SreeAyinampudi.Comparison of the antibacterial activity, volatiles and fatty acid composition of lipids of Phycopsis species collected at different locations from the Bay of Bengal (Orissa coast)Journal of the Serbian Chemical Society 2009 Volume 74, Issue 2, Pages: 133-139 
73. Gopalakrishnan, Karikalan; RajangamUdayakumar .GC-MS Analysis of Phytocompounds of Leaf and Stem of Marsileaquadrifolia (L.)International Journal of Biochemistry Research \& Review 4.6 (2014): 517-526

74. Faheem Amir*,Chemical Constituents and Biological Properties of the Marine Soft Coral Nephthea: A Review (Part 2)Tropical Journal of Pharmaceutical Research ,June 2012;11(3);499-517

75. Austin B. \& Austin D.A .(1989).Methods for microbial examination of fish and shellfish pp.317.Ellis Horwood Limited

76. LaDonswann\& M Randy White DVM:Dignosis\& Treatment of Aeromonashydrophila'Infection of fish'Aquaculture extension. Illinos-Indiana Sea grant Programme 